\title{
An International Registry of Granulocyte Transfusions
}

\author{
Monica B. Pagano ${ }^{a}$ Suzy Morton ${ }^{b}$ Claudia S. Cohn ${ }^{c}$ Sylvie Gross ${ }^{d}$ Jose Kutner ${ }^{\mathrm{e}}$ \\ Antoine Lewin ${ }^{f}$ Jeffrey McCullough ${ }^{c}$ Irwin Schweitzer ${ }^{g}$ Alan T. Tinmouth ${ }^{\mathrm{h}}$ Kamille West $^{\mathrm{i}}$ \\ Simon J. Stanworth ${ }^{\mathrm{j}}$ \\ a Laboratory Medicine, University of Washington Medical Center, Seattle, WA, USA; \\ ${ }^{b}$ NHS Blood and Transplant, University Hospitals Birmingham, Birmingham, UK; \\ ${ }^{c}$ Laboratory Medicine and Pathology, University of Minnesota Medical Center, Minneapolis, MN, USA; \\ ${ }^{\mathrm{d}}$ Etablissement Français du Sang, La Plaine-Saint Denis, France;

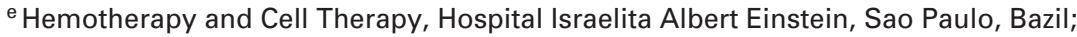 \\ ${ }^{f}$ Medical Affairs and Innovation, Héma-Québec, Montreal, QC, Canada; \\ ${ }^{9}$ Clinical Epidemiology Program Center for Transfusion Research, Ottawa Hospital Research Institute, Ottawa, ON, Canada; \\ h Departments of Medicine and Laboratory Medicine and Pathology, Ottawa Hospital Center for Transfusion Research, \\ Ottawa Hospital Research Institute, Ottawa, ON, Canada; \\ i Department of Transfusion Medicine, National Institutes of Health Clinical Center, Bethesda, MD, USA; \\ j NHS Blood \& Transplant/Oxford University Hospitals NHS Foundation Trust, and University of Oxford; Oxford, UK
}

\section{Keywords}

Granulocytes · Neutropenia · Infection · Registry

\section{Summary}

Introduction: Granulocyte transfusions are used to either treat or prevent life-threatening infections in neutropenic patients. Current evidence from clinical trials does not support or reject efficacy, nor guide practice. Methods: A group of investigators have led the efforts to create an online registry to gather information on granulocyte transfusion practices from as broad a range of international settings. The data forms were adapted from an ongoing study in England for electronic data management. Data is collected at the time of the request for granulocytes, weekly, at 28 days, and at 6 months. Information collected includes donor, granulocyte unit, patient and illness characteristics, and outcomes. Results: The PROspective GRanulocyte usage and outcomEs Survey (ProGrES) is currently open for data entry. Centres across the UK have collected data on 80 subjects. Five institutions from 4 countries ( 2 from the US, 1 each from Brazil, and national services in Canada and France) are in the process of joining the study. Other countries have expressed interest. Conclusion: It is feasible to develop an interna-

On behalf of BEST collaboration. tional registry of granulocyte transfusions to characterise current practices and describe outcomes. This registry would provide a platform to explore the relationship between intervention and outcomes, and to generate evidence to inform granulocyte transfusion efficacy.

(C) 2018 S. Karger GmbH, Freiburg

\section{Introduction}

Granulocyte transfusions have been collected and transfused over many decades to treat or prevent life-threatening infections in neutropenic patients. This practice is not widely accepted, since clinical efficacy has not been proven by randomised controlled trials (RCTs). Current evidence does not refute or support this practice, but for many clinicians and providers granulocyte transfusions are considered a valid attempt to treat a life-threatening infection until the patient recovers sufficient neutrophil counts or receives a haematopoietic progenitor cell transplant.

This review is intended to provide an overview of available granulocyte products, to describe the current evidence evaluating the clinical efficacy of granulocyte transfusions and to discuss the creation of an international registry of granulocyte transfusions describing current practices and evaluating outcomes.

\section{KARGER}

() 2018 S. Karger GmbH, Freiburg

Fax +497614520714 


\section{Granulocyte Products}

Granulocytes for transfusion can be obtained by one of two means: by apheresis, or as a component derived from whole blood donation. Apheresis granulocyte products are collected from donors stimulated with steroids (dexamethasone, prednisone) with or without the administration of granulocyte colony-stimulating factor (G-CSF) to increase the circulating granulocyte count prior to apheresis, enabling greater yields of granulocytes to be collected for transfusion. Overall, granulocyte units collected from donors without stimulation will contain 0.1 to $1 \times 10^{10}$ neutrophils, units from donors stimulated with steroids only will contain 1 to $2.5 \times 10^{10}$ neutrophils, and units collected from donors stimulated with steroids and G-CSF will contain approximately 4 to $8 \times 10^{10}$ neutrophils or higher depending on the G-CSF dose and administration schedule [1-4].

Apheresis granulocyte collections may be obtained from community donors or directed donors who are 'family and friends' of patients. The process involves multiple steps, including screening and counselling donors, infectious disease and serology testing, and donor stimulation with steroids alone or with G-CSF. All these first steps are usually accomplished during one or two appointments, with a subsequent appointment for the apheresis collection.

The apheresis collection is done several hours after the stimulation (frequently, the donor receives steroids alone or with G-CSF the night before of the morning collection), citrate is used as anticoagulant, and the procedure last approximately $2 \mathrm{~h}$. A sedimentation agent such as hydroxyethyl starch is used to facilitate the separation between red blood cells and granulocytes. Adverse effects related to the procedure are infrequent and usually mild and related to citrate toxicity. Side effects related to the use of a single dose of G-CSF include mild musculoskeletal pain, headache and fatigue [5]. In contrast, repeated doses of G-CSF typically used for stimulation of stem cell donors can result in more severe adverse effects, including splenomegaly and rarely splenic rupture, acute lung injury and flare of inflammatory diseases [6]. The unit is typically released from the collection facility to the hospital transfusion service soon after the collection to allow for secondary processing as indicated (irradiation, volume reduction) and transfusion within $24 \mathrm{~h}$ of the collection. Granulocyte units can be briefly stored at room temperature without agitation, but because neutrophil function and survival decline soon after the collection, the unit should be transfused with minimal delay.

Several other features need highlighting about this process. The yield of cells is more variable than might be expected, and sometimes poor. Availability of a licenced anticoagulant solution has caused issues e.g. in the UK. The process is very resource-intensive, often with requirements on top of an already stretched apheresis unit, committed to stem cell collections which cannot be readily moved to another time. It should be emphasised that this process is time-critical, often requiring planning at short notice for an apheresis team. Donors are difficult to recruit, and a sufficient number of donors are not always available to provide daily granulocyte transfusion support.
In contrast, a main source of granulocytes in the UK is derived from whole blood donations, which has been available for many years and has some immediate advantages of availability. These donations are commonly described as 'buffy coats' as they are derived from the buffy coat layer between red cells and plasma in centrifuged whole blood donations. In England, by providing a standard adult component derived from two pools of 10 donations, a consistent daily cell dose of around $2 \times 10^{10}$ cells may be transfused to patients. The characterisation of this pooled granulocyte component derived from whole blood donations have been described and involves the addition of platelet-additive solution but without the need for hydroxyethyl starch or dextran to sediment red cells during processing [7]. In addition to cell content, a range of in vitro tests for measures of neutrophil function were determined during storage [8]. The volume and red cell contamination of this product is vastly reduced compared to standard buffy coats and is similar to an apheresis granulocyte collection. The results for $\mathrm{pH}$, viability and neutrophil function indicated well maintained function during storage up to $24 \mathrm{~h}$, and some measures of neutrophil function were preserved for longer [9]. There were no statistically significant differences when this optimised granulocyte component was compared to either the standard buffy coat or fresh whole blood. Therefore, the method for producing a pooled granulocyte component derived from whole blood donations described above appears to provide granulocytes the in vitro function of which is maintained for up to $24 \mathrm{~h}$ of storage. This component has been evaluated in a clinical study and may have advantages of ready availability for transfusion on a daily basis if adequate whole blood donations have been collected the day before [10]. Ready access to units may be clinically important given that there is some evidence that provision of granulocytes at early onset of severe infection may be critical [11].

\section{Evidence}

Publications have reported transfusions of granulocytes in neutropenic patients both for therapeutic indications, when patients have an infection refractory to antimicrobials, or for secondary prophylaxis. [12-14]. Other studies with variable or promising, but overall inconclusive results have been undertaken both in adults and children [11, 14-16]. Overall, the existing literature remains heavily dominated by case reports and small case series, with many risks including publication bias. Those randomised trials that have been conducted include those completed over 20-30 years ago, when different guidelines, standards of care and infection management applied.

\section{Systematic Reviews of RCTs}

Potential efficacy including a dose-dependent effect has been raised by systematic reviews / meta-analyses, but the most recent Cochrane reviews find no overall evidence of a benefit and point to considerable uncertainty [17-20]. These reviews draw on many randomised studies conducted over a long period aimed at trying to establish effectiveness of granulocyte transfusions. 
The recently updated review on prophylaxis searched for all RCTs comparing patients receiving granulocyte transfusions with a control group receiving no granulocyte transfusions. Twelve trials were reported to meet the inclusion criteria; one trial was still ongoing, and 11 were eligible, for a total of 653 participants. Some trials were conducted over 30 years ago $(\mathrm{N}=9)$, many at times when very different standards of supportive and anti-microbial care existed by comparison to current practice. Ten studies included only adults, and two studies included children and adults [19]. All-cause mortality was reported for 9 studies (609 participants), although quantitative analysis was only reported across 7 studies, which reported no difference between patients receiving prophylactic granulocyte transfusions and those that did not (437 participants; RR 0.92, 95\% CI 0.63-1.36). For the outcome of mortality due to infection again there was no difference between groups. The reviewers reported a decreased number of patients with bacteraemia and fungaemia in the participants receiving prophylactic granulocyte transfusions ( 9 studies; 609 participants; RR $0.45,95 \%$ CI $0.30-0.65)$. Interestingly, there was a suggestion of dose effect for a decreased number of patients with infections over 30 days in the patients receiving prophylactic granulocyte transfusions in a higher-dose granulocyte group. However, overall the quality of the evidence was described as very low to low across the different outcomes according to GRADE methodology, due to many of the studies being at high risk of bias and many of the outcome estimates being imprecise.

A further update of a systematic review on treatment indications for granulocytes incorporated the recent RING trial [15]. The RING study is the largest RCT of granulocyte transfusions, in which 114 patients with neutrophil count $<0.5 \times 10^{9} / 1$ and proven or probable bacterial or fungal infection were included. As for the review of prophylactic trials, 10 RCTs met the inclusion criteria, for a total of 587 participants. Some studies were conducted over 30 years ago $(\mathrm{N}=8)$ [20]. Unfortunately, 3 studies re-randomised participants, and therefore quantitative analysis could not be performed to include these trials. There was no difference in allcause mortality over 30 days between participants receiving therapeutic granulocyte transfusions and those that did not (6 studies; 321 participants; RR 0.75 , 95\% CI 0.54-1.04). There were no clear differences in other outcomes, and there was insufficient data to report on risks of harm, e.g. a difference in serious pulmonary adverse events. Overall, it was not possible to establish whether granulocyte transfusions affect all-cause mortality. As for the prophylactic trials, again, the quality of the overall evidence was very low to low across different outcomes according to GRADE methodology.

Clearly, one message from these reviews was how the strengths of any conclusions were severely limited by the sample sizes and low methodological quality. With this in mind, it was reported in 1 review, that to be able to detect a decrease in all-cause mortality from $35 \%$ to $30 \%$ would require a study containing at least 2,748 participants ( $80 \%$ power, $5 \%$ significance). This appears unrealistic and highly expensive for trials with no clear source of funding.

\section{Next Steps and Background to Registries}

The current state of play needs to recognise two issues. First, granulocyte transfusions continue to be requested. Second, the evidence of effectiveness from RCTs is inconclusive and does not support nor exclude a role. Future robust RCTs evaluating efficacy of granulocyte transfusions are considered very difficult to undertake and unlikely to be funded. This is due to multiple difficulties including the sample sizes needed to conduct these studies (and their high costs), challenges in donor recruitment and providers' attitude to the research study (i.e. clinicians not willing to randomise their patients due to their preconceptions on efficacy or lack of efficacy). However, anecdotal evidence of benefit in selected patients from physicians in many countries continues to be well recognised, and this has generated groups of clinician 'believers', who feel that granulocyte transfusions may have a role in selected patients, although equally there are many 'non-believers' as well. For these reasons, we consider that observational studies, and especially registries, should now be considered to advance the field and as a valid alternative to conducting a RCT.

Registries can be developed for administrative (quality control, data trending) or research purposes (clinical practice, patient outcomes, safety and comparative effectiveness). Registries collect detailed information at patient, disease and therapeutic intervention levels. They are a particularly good methodological tool for lowfrequency diseases for which clinical trials would not be feasible. Since patients are not selected or excluded based on pre-stipulated protocols, surveys are true descriptions of 'real-world' clinical practice which allows for better generalisability of the results when compared to controlled trials. Large registries can inform practice variation, trends and outcomes, and can be designed with links to a bio-specimen repository.

Registries have also been utilised as the starting point for clinical studies, the 'registry-based randomised controlled trial' [21]. In this novel research methodology, patients are selected from a registry that contains patient and disease level information, allowing for rapid identification of subjects, enrolment, randomisation and follow-up. By this means, the registry provides a platform to facilitate the consecutive enrolment of patients resulting in increased efficiency and accrual, and overall to decrease costs [22]. Several registry-based randomised trials have been successfully used in different clinical settings, including the TASTE trial (comparing two therapeutic interventions for myocardial ischaemia), the CHAP trial (testing the effectiveness a health awareness programme), and the REDUCE MRSA trial (comparing two interventions for MRSA decolonisation) [23-25].

Registries have already been used for a variety of clinical situations and purposes. They are organised in a centralised repository, the Registry of Patient Registries (RoPR). The RoPR was established by the Agency of Healthcare Research and Quality (AHRQ) of the US Federal Department of Health and Human Services, with the purpose of improving transparency and reducing redundancy in registry-based research (https://patientregistry.ahrq.gov/faq/). More than 600 registries are identified when using 'cancer' as the 
search term, and no registries for granulocyte transfusions are currently registered.

It is important to recognise that registries have limitations. Quality and completeness of data entry remains paramount to the analyses and the value of a registry. Other concerns have been expressed regarding issues of patient consent and whether patients are always fully aware of the possible subsequent uses of their data. The major weakness in the proposed Registry is the lack of data on a non-transfused or control arm. To an extent this can be mitigated by collecting larger numbers of cases, supporting analyses to explore relative effectiveness of different intervention strategies (e.g. by dose of granulocytes). This is further strengthened by collection of consecutive patient data as far as possible.

\section{An International Registry of Granulocyte Transfu- sions - Details and Current State}

The primary outcome measure of the Registry is to describe how granulocytes are being used, in particular to characterise patient demographics, underlying conditions, infectious disease process (site, organism, therapeutic interventions), granulocyte treatment (frequency and dosage), donor and granulocyte unit features, and clinical outcomes.

Data is collected on all granulocyte products and, for patient data at the time of the request for granulocytes, weekly, at 28 days and at 6 months. Information collected include: i) granulocyte unit characteristics (type of donation (apheresis vs. buffy coat), cell count), and ii) donor information (donor demographics, CMV status, $\mathrm{ABO}$ type, number of donations, type and dose of mobilising agents and granulocyte apheresis absolute dose) and patient data (patient demographics, diagnosis, disease severity, duration of neutropenia, infection characteristics (infection site, microorganism, antimicrobial therapy), resolution of infection, mortality, CMV status, $\mathrm{ABO}$ type and alloimmunisation). Without specific exclusion criteria, data will be collected on all patients for whom granulocytes are authorised. This will allow a large heterogeneous population in the registry, leading to a greater external validity of the study and allowing estimation of subgroup differences in treatment effect.

The Registry arm in England, termed PROspective GRanulocyte usage and outcomEs Survey (ProGrES) (ProGrES@nhsbt.nhs.uk) has commenced. This started collecting information on indications, dose and outcomes following use of granulocytes administered to children and adults in spring 2017. The Registry is open to all institutions from all countries that volunteer to participate. Information will be collected on patients at all participating hospitals receiving pooled granulocytes or directed donations collected by apheresis. Individual patient consent was deemed by the NHS Health Research Authority not to be required, as the data were routinely available and fully anonymised.
Currently, 80 patients receiving granulocytes (or requests) have been identified and added to the database in the UK. At this early stage, all but 1 patient received a product derived from whole blood.

Through the Biomedical Excellence for Safer Transfusion (BEST) Collaborative, the Registry has been expanded to a webbased international collaborative involving multiple countries. It is anticipated that patients from other countries who receive granulocytes collected by apheresis or whole blood will be added to the Registry as centres in other countries join the study. Multiple blood collection centres and hospitals from different countries have indicated their willingness to participate. To join the Registry, hospitals or blood collection centres must obtain Institutional Review Board (IRB) or similar approval and complete a Data Use Agreement (DUA) with the sponsoring institution (Ottawa Health Research Institute, Ottawa, ON, Canada). After the indicated permissions are obtained, the investigator is granted access to the on-line Registry to upload patient information (The data entry front page can be found at https://omc.ohri.ca/ProGrES).

\section{Conclusion}

Clinical trials do not provide conclusive evidence to support, reject or guide granulocyte transfusion practices. Registries have demonstrated to be a useful tool to describe real-world practices, analyse trends and observe outcomes in different clinical settings. Based on our experience, the creation of an international registry of granulocyte transfusions is possible, represents a promising tool to characterise current practices, informs best granulocyte transfusion practices, and allows for comparative efficacy type analyses. The lessons learnt from this international collaborative initiative could be applied to other topics in transfusion medicine.

\section{Acknowledgements}

We recognise support and interest from a number of other individuals, institutions and countries, including Pierre Robillard, Gilles Delage, Andre Lebrun, Marc Germain, Benjamin Rioux-Massé (Quebec, Canada), Pierre Tiberghien, Claire Boulat (France), Mark Yazer, Mark Fung (USA), Ana Yokoyama (Brazil), James Daly, Peta Dennington, Frank Hong, David Irving, Jo Pink (Australia), Patrick Wuchter, Peter Bugert, Torsten Schulze (Germany), Tanja Netelenbos, Hans Vrielink (the Netherlands), Volker Witt (Austria), Richard Charlewood (New Zealand). We also wish to thank Dr Charlotte Brierley and NHSBT Clinical Trials Unit for supporting the initial planning of data collection processes and forms (Emma Laing, Chloe Fitzpatrick, Lorna Purkiss, Alison Deary, Renate Hodge, Ellie Curnow, Ana Mora).

\section{Disclosure Statement}

The authors did not provide a conflict of interest statement. 


\section{References}

1 Strauss RG, Hester JP, Vogler WR, Higby DJ, Snikeris AC, Imig KM, Greazel C, Mallard G, Burnett D, Gupta $\mathrm{S}$, et al: A multicenter trial to document the efficacy and safety of a rapidly excreted analog of hydroxyethyl starch for leukapheresis with a note on steroid stimulation of granulocyte donors. Transfusion 1986;26:258-264.

$\checkmark 2$ Bensinger WI, Price TH, Dale DC, Appelbaum FR, Clift R, Lilleby K, Williams B, Storb R, Thomas ED, Buckner CD: The effects of daily recombinant human granulocyte colony-stimulating factor administration on normal granulocyte donors undergoing leukapheresis. Blood 1993;81:1883-1888

3 Caspar CB, Seger RA, Burger J, Gmur J: Effective stimulation of donors for granulocyte transfusions with recombinant methionyl granulocyte colony-stimulating factor. Blood 1993;81:2866-2871.

4 Liles WC, Rodger E, Dale DC: Combined administration of G-CSF and dexamethasone for the mobilization of granulocytes in normal donors: optimization of dosing. Transfusion 2000;40:642-644.

5 Price TH, Bowden RA, Boeckh M, Bux J, Nelson K, Liles WC, Dale DC: Phase I/II trial of neutrophil transfusions from donors stimulated with G-CSF and dexamethasone for treatment of patients with infections in hematopoietic stem cell transplantation. Blood 2000; 95:3302-3309.

6 McCullough J, Kahn J, Adamson J, Anderlini P, Benjamin R, Confer D, Eapen M, Hirsch B, Kuter D, Lazarus E, Pamphilon D, Stroncek D, Sugarman J, Wilson R: Hematopoietic growth factors - use in normal blood and stem cell donors: clinical and ethical issues. Transfusion 2008;48:2008-2025.

7 Bashir S, Stanworth S, Massey E, Goddard F, Cardigan $\mathrm{R}$ : Neutrophil function is preserved in a pooled granulocyte component prepared from whole blood donations. Br J Haematol 2008; 140:701-711.

8 Bashir S, Cardigan R: Granulocyte concentrates: how can we assess their quality? Transfus Med 2003; 13: 245-257.

9 Schwanke U, Schrader L, Moog R: Storage of neutrophil granulocytes (PMNs) in additive solution or in autologous plasma for $72 \mathrm{~h}$. Transfus Med 2005;15:223-231.

10 Massey E, Harding K, Kahan BC, Llewelyn C, Wynn R, Moppett J, Robinson SP, Green A, Lucas G, Sadani D, Liakopoulou E, Bolton-Maggs P, Marks DI, Stanworth S: The granulocytes in neutropenia 1 (GIN 1) study: a safety study of granulocytes collected from whole blood and stored in additive solution and plasma. Transfus Med 2012;22:277-284.
11 Sachs UJ, Reiter A, Walter T, Bein G, Woessmann W: Safety and efficacy of therapeutic early onset granulocyte transfusions in pediatric patients with neutropenia and severe infections. Transfusion 2006;46:1909-1914.

12 Hubel K, Carter RA, Liles WC, Dale DC, Price TH, Bowden RA, Rowley SD, Chauncey TR, Bensinger WI, Boeckh M: Granulocyte transfusion therapy for infections in candidates and recipients of HPC transplantation: a comparative analysis of feasibility and outcome for community donors versus related donors. Transfusion 2002;42:1414-1421.

13 Kerr JP, Liakopolou E, Brown J, Cornish JM, Fleming D, Massey E, Oakhill A, Pamphilon DH, Robinson SP, Totem A, Valencia AM, Marks DI: The use of stimulated granulocyte transfusions to prevent recurrence of past severe infections after allogeneic stem cell transplantation. Br J Haematol 2003;123:114-118.

14 Oza A, Hallemeier C, Goodnough L, Khoury H, Shenoy S, Devine S, Augustin K, Vij R, Trinkaus K, Dipersio JF, Adkins D: Granulocyte-colony-stimulating factor-mobilized prophylactic granulocyte transfusions given after allogeneic peripheral blood progenitor cell transplantation result in a modest reduction of febrile days and intravenous antibiotic usage. Transfusion 2006;46:14-23.

15 Price TH, Boeckh M, Harrison RW, McCullough J, Ness PM, Strauss RG, Nichols WG, Hamza TH, Cushing MM, King KE, Young JA, Williams E, McFarland J, Holter Chakrabarty J, Sloan SR, Friedman D, Parekh S, Sachais BS, Kiss JE, Assmann SF: Efficacy of transfusion with granulocytes from G-CSF/dexamethasonetreated donors in neutropenic patients with infection. Blood 2015;126:2153-2161.

16 Seidel MG, Peters C, Wacker A, Northoff H, Moog R, Boehme A, Silling G, Grimminger W, Einsele H: Randomized phase III study of granulocyte transfusions in neutropenic patients. Bone Marrow Transplant 2008; 42:679-684.

17 Vamvakas EC, Pineda AA: Meta-analysis of clinical studies of the efficacy of granulocyte transfusions in the treatment of bacterial sepsis. J Clin Apher 1996;11: 1-9.

18 Vamvakas EC, Pineda AA: Determinants of the efficacy of prophylactic granulocyte transfusions: a metaanalysis. J Clin Apher 1997;12:74-81.
9 Estcourt LJ, Stanworth S, Doree C, Blanco P, Hopewell S, Trivella M, Massey E: Granulocyte transfusions for preventing infections in people with neutropenia or neutrophil dysfunction. Cochrane Database Syst Rev 2015;6:CD005341.

20 Estcourt LJ, Stanworth SJ, Hopewell S, Doree C, Trivella M, Massey E: Granulocyte transfusions for treating infections in people with neutropenia or neutrophil dysfunction. Cochrane Database Syst Rev 2016; 4:CD005339.

21 Lauer MS, D’Agostino RB Sr: The randomized registry trial - the next disruptive technology in clinical research? N Engl J Med 2013;369:1579-1581.

22 Li G, Sajobi TT, Menon BK, Korngut L, Lowerison M, James M, Wilton SB, Williamson T, Gill S, Drogos LL, Smith EE, Vohra S, Hill MD, Thabane L; Symposium on Registry-Based Randomized Controlled Trials in Calgary: Registry-based randomized controlled trials what are the advantages, challenges, and areas for future research? J Clin Epidemiol 2016;80:16-24.

23 Frobert O, Lagerqvist B, Olivecrona GK, Omerovic E, Gudnason T, Maeng M, Aasa M, Angeras O, Calais F, Danielewicz M, Erlinge D, Hellsten L, Jensen U, Johansson AC, Karegren A, Nilsson J, Robertson L, Sandhall L, Sjogren I, Ostlund O, Harnek J, James SK; TASTE Trial: Thrombus aspiration during ST-segment elevation myocardial infarction. N Engl J Med 2013;369:1587-1597.

24 Kaczorowski J, Chambers LW, Karwalajtys T, Dolovich L, Farrell B, McDonough B, Sebaldt R, Levitt C, Hogg W, Thabane L, Tu K, Goeree R, Paterson JM, Shubair M, Gierman T, Sullivan S, Carter M: Cardiovascular Health Awareness Program (CHAP): a community cluster-randomised trial among elderly Canadians. Prev Med 2008;46:537-544.

25 Huang SS, Septimus E, Kleinman K, Moody J, Hickok J, Avery TR, Lankiewicz J, Gombosev A, Terpstra L, Hartford F, Hayden MK, Jernigan JA, Weinstein RA, Fraser VJ, Haffenreffer K, Cui E, Kaganov RE, Lolans K, Perlin JB, Platt R; CDC Prevention Epicenters Program; AHRQ DECIDE Network and Healthcare-Associated Infections Program: Targeted versus universal decolonization to prevent ICU infection. N Engl J Med 2013;368:2255-2265. 